\title{
A RAPID ONE POT SYNTHESIS OF NITRILES FROM ALDEHYDES AND HYDROXYLAMINE HYDROCHLORIDEIN PRESENCE OF SODIUM CHLORIDE
}

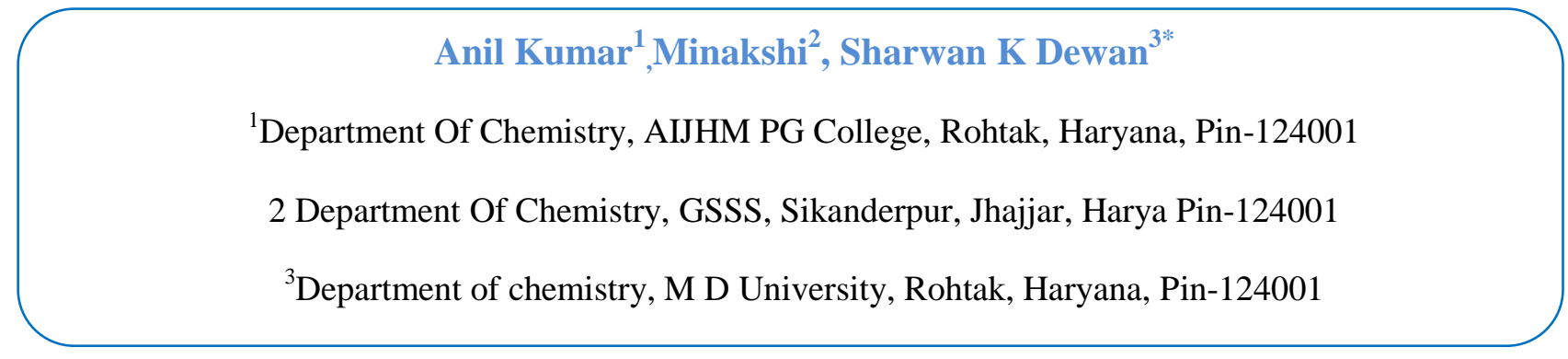

\section{ABSTRACT:}

Sodium chloride was used as a highly efficient catalyst for a rapid one pot synthesis of nitriles from aldehydes and hydroxylamine hydrochloride in $85-93 \%$ yields.

\section{Graphical Abstract}

$\mathrm{RCHO}+\mathrm{H}_{2} \mathrm{NOH} . \mathrm{HCl}$

\section{$\rightarrow \quad \mathrm{RCN}$}

\section{SODIUM CHLORIDE}

KEYWORDS : Nitriles, Aldehydes, Hydroxylamine hydrochloride,Sodium chloride.

\section{INTRODUCTION}

Nitriles occupy a significant position among functional groups in organic chemistry.[1] In fact, they serve as important intermediates in the synthesis of pharmaceuticals, agricultural chemicals, dyes, material sciences as well as in microbial metabolism. Further, they are viable precursors for the synthesis of a variety of nitrogen containing functional compounds. They can be transformed into a number of functional moieties like primary amines upon reduction, amides or carboxylic acids upon hydrolysis, ketones by reaction with organometallics etc. Nitriles are also widely used in petrochemical industry.

$$
\mathrm{RCHO}+\mathrm{H}_{2} \mathrm{NOH} . \mathrm{HCl}
$$

\section{SODIUM CHLORIDE $\quad$ 85-93\%}

Nitriles can be synthesized by the reaction of alkyl halides with metal cyanides but the reaction is inconvenient because of the toxicity of the cyanide substrates. Other synthetic methods include the dehydration of oximes and aldoximes. The dehydration of aldoximes has been achieved by using reagents like trimethylamine/sulfurdioxide, sulfurylchloride, sulfurylchloridefluoride etc..[2]Recently we reported a one pot synthesis of nitriles from aldehydes and hydroxylamine hydrochloride under microwave irradiation by using 
sodium sulfate and sodium bicarbonate.[3-4]We now report herein a rapid one pot synthesis from aldehydes and hydroxylamine hydrochloride by using an inexpensive readily accessible sodium chloride.

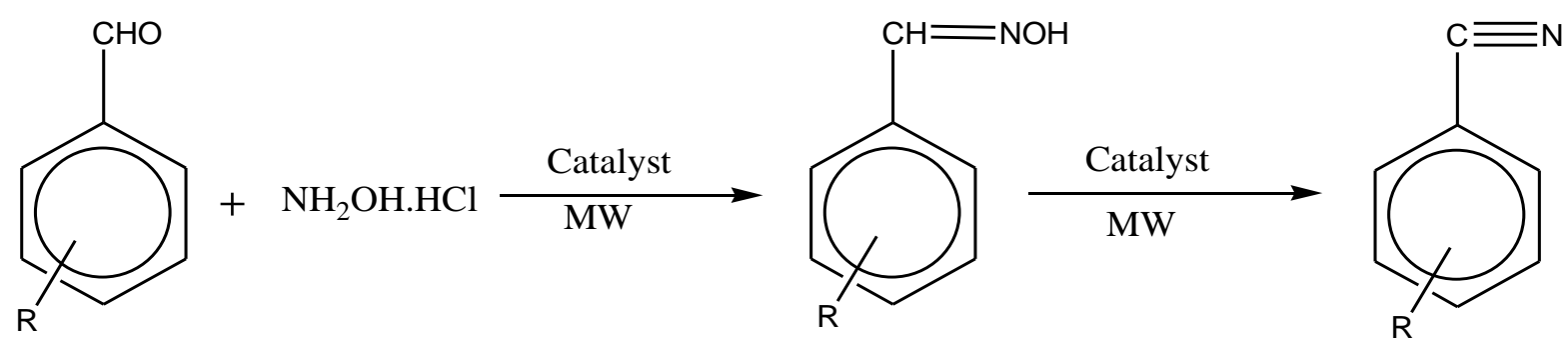

\section{RESULTS AND DISCUSSION}

Studies were initiated with p-hydroxybezaldehyde as that is a most readily available in pure form. The mixture of this aldehyde and hydroxylaminehydrochloride taken in 1:1 molar ratio was mixed with sodium chloride and microwaved at a range of temperatures but $50^{\circ} \mathrm{C}$ was found to be optimum temperature when it afforded the desired nitrile in $90 \%$ yield. Therefore, subsequent reactions were carried out at this temperature. A number of variously substituted arylaldehydes, and aliphatic aldehydes and alpha-beta unstuarated aldehydes were converted into corresponding nitriles.Thus were synthesized 4 - hydroxy benzonitrile, 2Hydroxybenzonitrile3,4-Dimethoxybenzonitrile, 3,4,5-Trimethoxybenzonitrile, 4-Methylbenzonitrile, 4Nitrobenzonitrile, 2-Nitrobenzonitrile, Decylnitrile, Octylnitrile, Citronellalnitrile,Salicylonitrile Transcinnamonitrile.The yields of the products are recorded in Table1. TLC was used to monitor the reactions .All the products were unequivocally identified by their spectroscopic and physical data.As can be seen from table 1 , the variously substituted and sterically hindered aldehydes all gave the corresponding nitriles.And the products were obtaind in high yields of $85-93 \%$.

Therefore, the newly developed rapid one pot method is very efficient and cost effective and the catalyst used is readily available in pure form, and is inexpensive as well and should find wide application.

TABLE 1: Synthesis of nitriles from aldehydes and hydroxylamine hydrochloride in presence of sodium chloride under microwave irradiation.
Entry
Product
Catalyst

yield $\%$ Time

1

2

2

2-Hydroxybenzonitrile
92

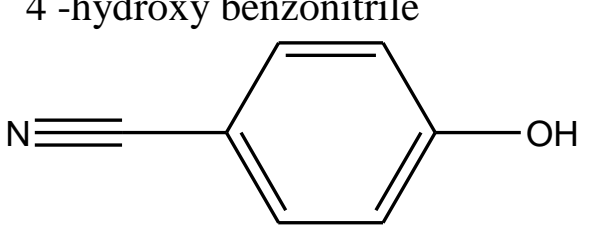

4 hydroxy benzonitrile

\section{4}




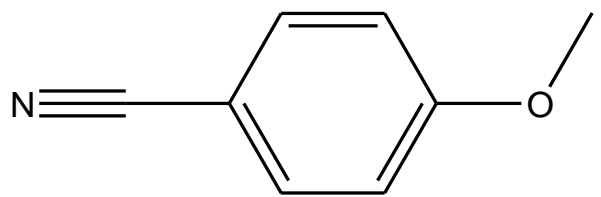

4-methoxybenzonitrile

4

3,4-Dimethoxybenzonitrile<smiles>COc1ccc(C#N)cc1OC</smiles>

3,4 dimethoxy benzonitrile

5

3,4,5-Trimethoxybenzonitrile91

4-Methylbenzonitrile

915

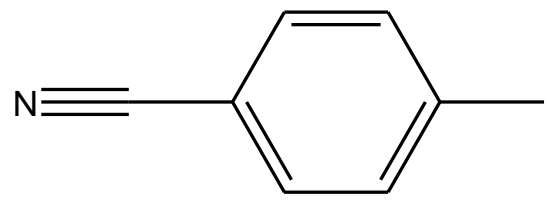

4 methyl benzonitrile

74-Nitrobenzonitrile

864

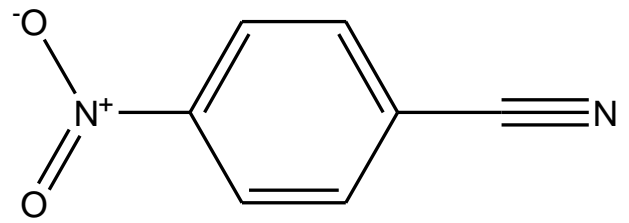

4-nitrobenzonitrile

8

2-Nitrobenzonitrile

854

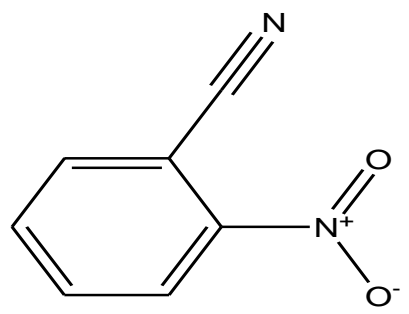

2-nitrobenzonitrile 


\section{9

10Octylnitrile

11Citronellalnitrile

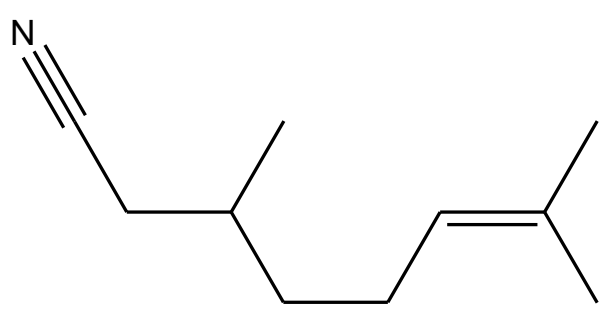

citronellonitrile

12 Salicylonitrile

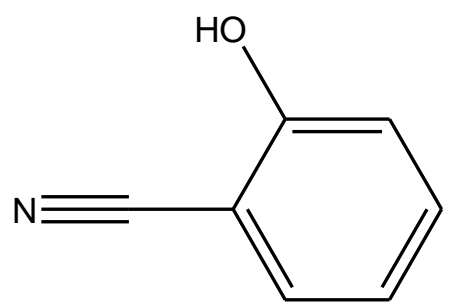

salicylonitrile

13

Trans-cinnamonitrile

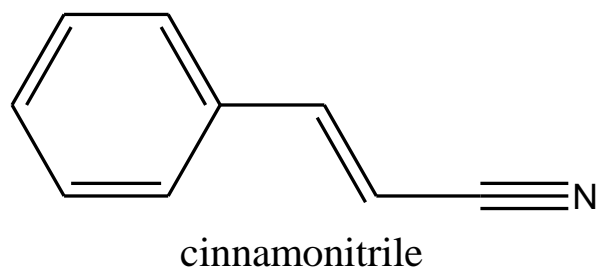

90

4

\section{4}

865

88

3.5

\section{MATERIALS AND METHODS}

Melting points are uncorrected and were determined by open capillary methods. The proton $\mathrm{nmr}$ spectra were recorded on a 400MHz NMR Spectrometer . All chemical shifts are expressed in parts per million with respect to tetramethylsilane(TMS) and in $\mathrm{CDCl}$. The IR spectra were obtained on a FT Nicolet Instrument.

In a typical procedure, 4-hydroxybezaldehyde ( $122 \mathrm{mg}, 1 \mathrm{mmol})$, hydroxylamine hydrochloride (105mg, $1.2 \mathrm{mmol}$ ) and sodium chloride were intimately mixed together in a five $5 \mathrm{~mL}$ Pyrex beaker and then irradiated at $50^{\circ} \mathrm{C}$ for $4 \mathrm{~min}$ when the reaction was found to be complete as shown by TLC. The mixture was extracted into dichloromethane $(25 \mathrm{~mL})$, the solution was filtered off and the solvent 
DOI : https://dx.doi.org/10.26808/rs.ph.i8v1.05

International Journal of Pharmaceutical Science and Health

Issue 8, Vol.1 (January-February 2018)

Available online on http://www.rspublication.com/ijphc/index.html

ISSN $2249-5738$

evaporated off to give a residue. The crude product was purified by column chromatography (benzene:ethylacetate, $4: 1$ ) to give the desired product.

\section{REFERENCES}

1 G A Olah, Y D Vankar, Synthesis, 1978,702

2 G A Olah, Narang S C , A Garcia-Luma, Synthesis, 1980,659

3 S K Dewan, R Singh, A Kumar, Synth Commun. 2003, 33, 3085

4S K Dewan, R Singh, A Kumar, Arkivoc, 2006,2,41.

5 Punam, A Kumar, SK Dewan, J Applicable Chemistry, 2014, 3, 639 